\title{
Instrumentos e estratégias de avaliação para mediação cultural
}

\author{
Julia Rocha Pinto* \\ Universidade do Estado de Santa Catarina
}

\begin{abstract}
Resumo
Este texto reflete sobre formas de avaliar o trabalho educativo que museus desenvolvem com seus públicos. Contemporaneamente, em resposta aos preceitos de mediação cultural que têm sido empregues nos museus, as propostas de avaliação se identificam como reflexões sobre os processos, recorrendo a diferentes instrumentos para aferir suas relações com os visitantes, nomeadamente: questionários, entrevistas, observações e mapas conceituais ou de sentido. A combinação e associação destas ferramentas é empregue por diferentes modelos que serão discutidos ao longo do texto, são eles: Generic Learning Outcomes, Assessment for Learning, Personal Meaning Maps e Contemporary Gallery Education. A análise de cada um dos modelos é realizada com exemplos práticos, visando identificar aspectos importantes para a construção de estratégias de avaliação para a mediação cultural.
\end{abstract}

\section{Palavras-chave}

Ação educativa; avaliação; museus de arte; ferramentas; reflexão.

\begin{abstract}
This text reflects on ways to evaluate the educational work that museums develop with their audiences. Contemporaneously, in response to cultural mediation precepts that have been employed in museums, proposals for evaluation are identified as reflections on the processes, using different instruments to assess their relationship with visitors, including: questionnaires, interviews, observations and conceptual or sense maps. The combination and association of these tools are used by different models that will be discussed throughout the text: Generic Learning Outcomes, Assessment for Learning, Personal Meaning Maps and Contemporary Gallery Education. The analysis of each of the models is carried out with practical examples, identifying important aspects to building assessment strategies for cultural mediation.
\end{abstract}

\section{Key-words}

Learning department; evaluation; art museums; tools; reflection.

\footnotetext{
* Julia Rocha Pinto é Doutora em Educação Artística pela Universidade do Porto e Mestre em Artes pela Universidade Estadual Paulista. Professora do Centro de Artes da Universidade do Estado de Santa Catarina. Realiza pesquisa sobre mediação cultural, relações entre museus e escolas e avaliação de propostas educativas no campo das artes visuais.
} 
O exercício educativo realizado pelos museus e instituições culturais está constantemente recriando novas formas de pensar a recepção de seus públicos, desenvolvendo práticas cada vez mais dialógicas e participativas. Variadas nomenclaturas têm sido utilizadas para designar o educador de museus e novos paradigmas caracterizam a mediação cultural. Diante das novas abordagens que vão sendo incorporadas na prática do educador de museus, diferentes perspectivas de pensar a avaliação surgem em resposta.

$\mathrm{Na}$ medida em que os setores educativos dos museus desenvolvem um trabalho colaborativo com seus públicos, construindo propostas mais dialogadas e construídas a partir dos múltiplos repertórios, as estratégias de avaliar o que realizam acompanham estes preceitos, buscando serem mais construtivas e reflexivas. Este texto ${ }^{1}$ discorre sobre quatro diferentes estratégias de avaliação que são recorrentemente empregadas para refletir e avaliar o trabalho educativo realizado em museus.

Primeiramente, para pensar as formas de reconsideração dos exercícios educativos, cabe conceber que os estudos de avaliação e pesquisa em instituições culturais são usados com diferentes propósitos, pensando em fornecer informações a fim de qualificar os serviços em desenvolvimento e oferecendo um retorno sobre o impacto das ações. Diante dos objetivos definidos para as avaliações - razões econômicas, resposta à instituição, crescimento do trabalho, pesquisas encomendadas - os estudos realizados podem assumir variadas formas, refletindo as escolhas tomadas no desenho das propostas e evidenciando que resultados se almejam com as ações educativas.

Compreender que paradigmas existem envolvendo a ideia de avaliação oferecerá ferramentas para repensar inclusivamente a prática educativa que se constrói, mormente porque as abordagens não são excludentes umas das outras e muitas vezes se sobrepõem no cotidiano dos serviços educativos, podendo influenciar a ação como um todo. Sobre esta riqueza de possibilidades, George Hein (1999: 307) refere "All forms of evaluation can be useful. The styles of evaluation available may appear to be so different that they are contradictory, but each can be use in certain situation".

Entender como os processos decorrem, conhecendo uma gama de possíveis formas e posicionamentos para refletir sobre as práticas, instrumentaliza a relação educativa oferecida pelos museus. Posteriormente, pode haver até mesmo a desconstrução destas abordagens e novos procedimentos serem criados de acordo com o contexto e os sujeitos. Neste sentido, apresentam-se

\footnotetext{
${ }^{1} \mathrm{O}$ presente texto é parte da tese "Reflexões sobre o meio - O espaço entre a Escola e o Museu de Arte Contemporânea", desenvolvida no Doutorado em Educação Artística da Universidade do Porto em 2015. 
técnicas de avaliação, registro de escuta e abordagens criadas e desenvolvidas no campo museal.

Os sistemas de avaliação existem como modelos cabíveis que são orientadores de uma experiência, mas, em cada processo, é adequado incluir diferentes modos de repensar as práticas. Ainda de acordo com Hein (1999: 307), "The choice of the form of evaluation that will be carried out depends on the problem that is being addressed, the audience to whom the work is directed, and the professional and personal inclinations of the people involved".

Começa-se, portanto, a partir de Phil Bull (1999: 295) propõe três categorias de avaliação: "front-end analysis, formative evaluation and summative evaluation". Estas três categorias podem ser compreendidas mais como momentos da avaliação e não são excludentes das demais. A primeira delas é uma etapa diagnóstica, que tenta identificar os problemas que podem vir a acontecer. A categoria formativa é relacionada com o trabalho em processo, que pode ser compreendido como um "teste de desenvolvimento", segundo 0 autor refere. Já a categoria somativa pretende tratar do que acontece durante o processo e que se constrói nas mudanças elaboradas.

A maior parte dos museus se dedica mais a esta última categoria, quando realiza procedimentos de avaliação depois que as ações foram finalizadas, como menciona Eilean Hooper-Greenhill (1994: 78). Todavia, estas três categorias, como mencionado previamente, não precisam ser substitutivas e devem ser até mesmo encaradas como diferentes etapas de um mesmo processo.

Neste sentido, pode-se pensar inclusive que a avaliação front-end pode acontecer no momento do acolhimento de uma visita educativa no museu, quando se começam a criar relações e a conhecer de que tipo de repertórios se trata naquele grupo ali presente, que perfil os participantes têm, que interesses trazem para a ação. Sobre esta categoria, Andrew J. Pekarik diz que:

Front-end evaluation that attempts to uncover "what people know" about a subject in order to refine the cognitive message that is to be the exhibition's objective will have trapped itself within the model of outcome-based development, since it is likely to ignore - or not solicit, or not notice - data that points to the value of other, very different visitor goals, including those that are unrelated to ideas. (2010: 108)

Reforça-se desta maneira a ideia de que a avaliação é parte de todo 0 processo e mesmo durante o início da mediação cultural, quando as relações de troca passam a ser estabelecidas, já existem processos de reflexão em curso. A avaliação processual se designa de diferentes formas e vai se 
executando com a adoção de distintas estratégias; escolher a mais efetiva maneira de convidar à participação dos sujeitos, engajando-os a refletir conjuntamente e repensar a prática é uma questão que envolve desafios.

Outro momento de avaliação é ainda adicionado por Nancy Cutler (2009: 119), que diz que, além destes três, existe também a "remedial evaluation", que busca reparar e configurar de outra forma os processos. Para a autora a preocupação neste momento é "What needs to be fixed to make it work better?". Desta forma, atua-se no sentido de repensar as práticas e até mesmo as formas de se avaliar. Cutler (2009: 119) indica que "This is different from summative evaluation because it presumes an expectation of making modifications".

Uma problemática identificada recorrentemente nos museus é que a avaliação se relaciona diretamente com investigação, sendo uma prática que requer ímpeto de pesquisa por parte da equipe que compõe o setor educativo. $\mathrm{Na}$ prática, contudo, o que se percebe é que nem todos os educadores estão engajados nesta procura de repensar suas práticas, uma vez que para alguns elementos aquele trabalho se constitui como um vínculo temporário, sem haver comprometimento com a pesquisa que é vista como necessária como impulso para encontrar efetivas formas de refletir sobre os processos. A este respeito, Hein (1999a: 307) considera que "No matter what the form of evaluation chosen, it is crucial that museum program staff be involved in the evaluation", problemática vivida na prática.

Esta motivação em relação ao caráter de investigação apresenta-se como proposta para todas as áreas de gestão dos museus, não se restringindo aos serviços educativos, e neste sentido Maria Economou (2004: 40) ainda sugere que deve haver uma formação para que todos os profissionais da equipe estejam aptos e envolvidos no processo de avaliação. Outra questão também importante é compreender, além da motivação, se os sujeitos têm disponibilidade para se envolverem neste processo reflexivo. Com essas premissas atendidas e com uma efetiva participação, ao menos da equipe constituinte do museu, passam-se a delinear estratégias diferentes que conduzem à produção de métodos próprios para cada contexto.

Em pesquisas realizadas ao longo da formação acadêmica (Pinto, 2012; 2015) tem-se encontrado sobretudo uma forma empregada pelos museus como meio para avaliar suas práticas, os questionários. $O$ uso de questionário como prática de avaliação é o mais difundido nos museus, que habitualmente incorporam questões de múltipla escolha para que seus visitantes qualifiquem a experiência na instituição. 
Um estudo realizado por George Binks e David Uzzell (1999: 298) que procura definir as potencialidades e fragilidades das técnicas usadas em processos de avaliação aponta os questionários como ferramentas positivas no sentido da facilidade de aplicação, uma vez que eles podem ser preenchidos em larga escala após sua elaboração; e de baixo custo, visto que os questionários fazem uso de materiais habitualmente presentes nas instituições. Contudo, os autores determinam que a fragilidade desta técnica está na forma como são elaborados, quando muitas vezes são mal desenvolvidos e não conseguem dar conta das particularidades dos processos. Além disso, apontam também como um aspecto negativo a demanda de trabalho que estes materiais geram posteriormente, com o processamento dos dados e leitura dos resultados.

A quantidade de público possível de atender e a possibilidade de realizar amostragem tornam os questionários a ferramenta mais prática de ser adotada, efetivamente; mas a esta facilidade de ampliação juntam-se questões de ordem conceitual que não dão conta do que acontece nos meandros. Uma fragilidade observada nesta recorrência é que por meio dos questionários a escuta é feita unidirecionalmente e qualquer possibilidade de resposta fica dificultada pelo intervalo temporal que pode acontecer se o museu levar a cabo este exercício e decidir tornar os resultados verdadeiramente efetivos.

Além dos questionários, outra estratégia também utilizada como prática de avaliação são as entrevistas. Retomando o estudo feito por Binks e Uzzell (1999: 299), como potencialidades destes instrumentos são apontados: 0 detalhamento qualitativo da informação que se aproxima dos desenvolvimentos dos processos, a exploração de resultados objetivados pelas partes interessadas e a dispensa da necessidade do uso de tecnologias digitais.

Em contrapartida, na mesma pesquisa, pensando nas fragilidades da ferramenta, Binks e Uzzell (1999: 299) consideram que as entrevistas tomam um tempo muito grande, conseguem dar conta de questões muito pontuais que podem não ser representativas de uma amostragem, necessitam da habilidade de entrevista por parte das equipes e também trazem a dificuldade de interpretação dos dados obtidos.

Outras técnicas de avaliação são as observações e os mapas de significados ou comportamentais. Binks e Uzzell (1999: 300) indicam como ponto positivo destas análises a aproximação aos sujeitos envolvidos nos processos educativos, porque, segundo os autores, com estas técnicas consegue-se verdadeiramente compreender as impressões e ver como as relações são criadas no espaço do museu. Outra potencialidade apontada neste caso diz respeito à possibilidade de usar outras técnicas como complemento, pensando 
os questionários e as entrevistas como desdobramentos do trabalho de observação ou de criação de mapas.

Para todas estas ferramentas de avaliação o tempo é sugerido como um indicativo negativo de sua aplicação, visto que estas formas de repensar as práticas exigem um trabalho longo no desenvolvimento e também na posterior leitura dos materiais produzidos. A interpretação é também apresentada como problemática, uma vez que tanto a produção destes relatos de observação como a criação de mapas conceituais se constrói com base em discursos desenvolvidos individualmente.

Tendo em conta algumas possibilidades de técnicas que são, habitualmente, utilizadas para se avaliarem as práticas educativas nos museus, pensa-se agora em métodos e abordagens que são criados e que se utilizam da combinação destas e de outras ferramentas. É difícil falar de modelos de avaliação dentro dos museus sem cair no risco de generalizar e apresentar como ideias universais de formações que emergem de condições socioculturais, econômicas e educativas. Consciente deste desafio e das limitações que se impõem, apresentam-se abordagens que têm sido construídas visando conhecer o domínio do campo.

Contemporaneamente têm sido criadas diferentes linhas para se pensar a avaliação em museus e até mesmo copiadas ou adaptadas por outras instituições. Neste sentido, serão apresentados quatro diferentes modelos para se reconhecerem possíveis práticas de reflexão dentro do museu: Generic Learning Outcomes, Assessment for Learning, Personal Meaning Maps e Contemporary Gallery Education.

A primeira delas partiu do Research Centre for Museums and Galleries (RCMG), da Universidade de Leicester, que desenvolveu para o órgão britânico Museums, Libraries and Archives Council (MLA) uma pesquisa de impacto de aprendizagem a fim de identificar processos e pensar em formas para avaliar a aprendizagem nestes espaços. A partir dessa pesquisa foram desenvolvidos os Generic Learning Outcomes, em reconhecimento do recente interesse pelos diferentes modos de aprendizagem que atuam de uma maneira flexível e pensando nos sujeitos como centro das práticas, tal como indicam Penny Jones, Libby Anson e Alex Hitchins (2008: 29).

A concepção dos Generic Learning Outcomes foi realizada dentro do programa Inspiring Learning for All ${ }^{2}$, que tem como objetivo avaliar os pontos fortes das instituições de cunho educativo e cultural, buscando melhorias no plano de trabalho, fornecendo evidências do impacto e melhorando o desempenho. Sua

\footnotetext{
2 Inspiring Learning for All é um programa do governo britânico Museum, Libraries and Archives Council, que propõe estratégias de avaliação para instituições de cunho cultural e educativo. 
criação se baseou em um modelo teórico onde existe o entendimento de que a aprendizagem ocorre na relação entre processos de identidade individuais e visões de mundo.

Esta abordagem refere que mesmo que os processos sejam individuais e que cada sujeito crie suas relações, a aprendizagem ainda pode ser agrupada em categorias genéricas. Relacionada com preceitos educativos já discutidos anteriormente, "The interpretive framework offered by the GLOs was based on constructivist and socio-cultural learning theory", diz Hooper-Greenhill, que ainda completa:

They consist of a conceptual framework for developing research questions, designing research tools and analysing and interpreting research findings that is particularly appropriate to the multi-dimensional, creative and open-ended learning environment of the museum and other cultural organisations.(2007: 60)

Os GLOs proporcionam a oportunidade de se criarem registros usando uma variedade de métodos de pesquisa, funcionando como uma estrutura interpretativa para os resultados produzidos dentro dos museus. A unidade destes resultados também permite uma circulação entre eles, dando a conhecer o material que é realizado para as exposições e que tem por hábito servir apenas para reflexão das próprias instituições.

As cinco categorias propostas por esta abordagem são: conhecimento e compreensão; habilidades; atitudes e valores; divertimento, inspiração e criatividade; e ação, comportamento e progressão. Estas categorias não têm hierarquia entre si e são compreendidas como focos por onde se podem construir relações profícuas para se repensarem as práticas educativas museais.

Dentro desta abordagem, de acordo com o site da Inspiring Learning for Al/3, a aprendizagem é um processo de engajamento ativo com a experiência e os processos podem envolver desenvolvimento de competências, conhecimento, compreensão, valores, ideias e sentimentos. Desta forma, se propõe uma aprendizagem com sentido na mudança e na vontade de aprender mais.

Como propostas de objetivos desta abordagem coloca-se que deve haver uma visão compartilhada de seu papel e propósito, com iniciativa, trabalho de equipe e posturas flexíveis, visando o desenvolvimento pessoal e profissional com abertura a novas ideias e abordagens. Esta construção se dá por meio da elaboração de processos de avaliação com base nas categorias propostas.

${ }^{3}$ Site do programa: http://inspiringlearningforall.gov.uk. 


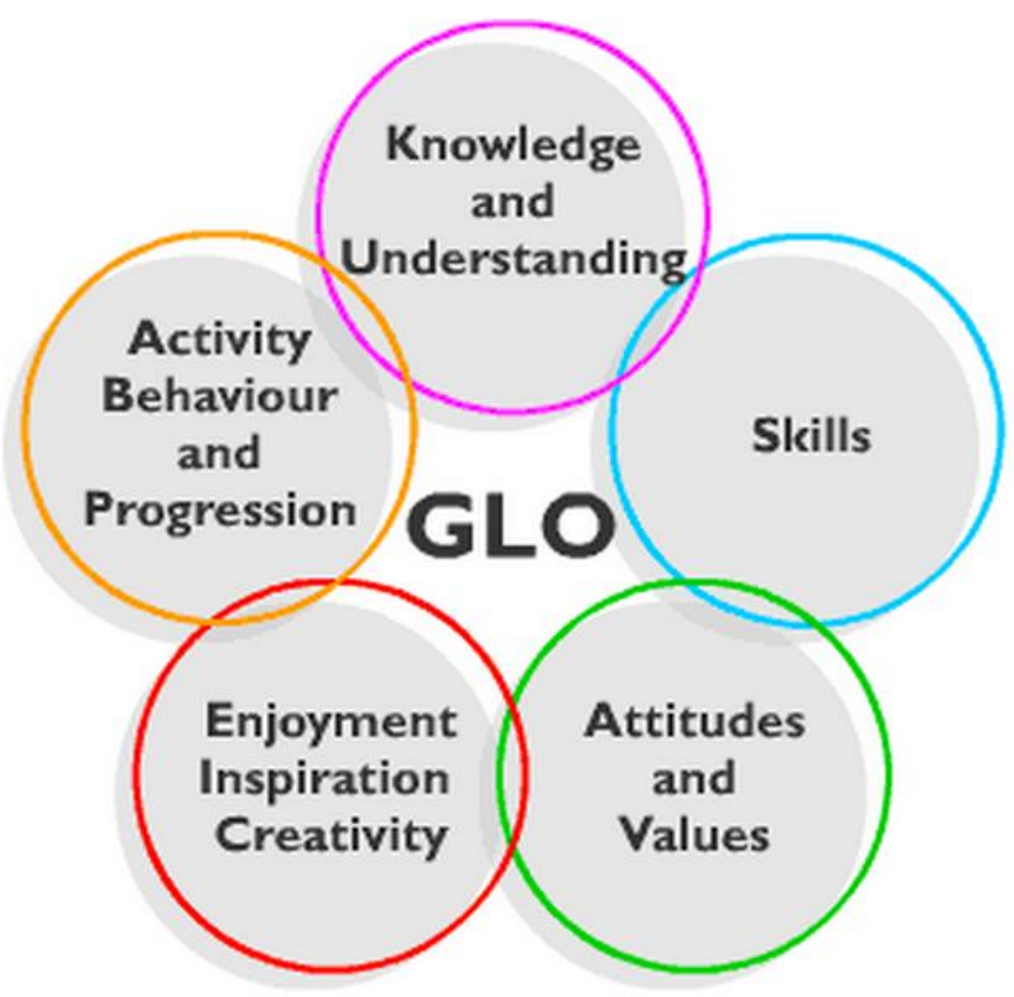

Fig. 1: Esquema dos Generic Learning Outcomes. Fonte: Site Inspiring Learning for All. Disponível em: <http://inspiringlearningforall.gov.uk/toolstemplates/genericlearning/index.html>. Acesso em: 8 Jun. 2015.

É importante salientar, como lembram Jones, Anson e Hitchins (2008: 29) que os GLOs foram concebidos como um ponto de partida para desenvolver uma análise mais detalhada dos objetivos de aprendizagem nos espaços culturais. 0 método foi desenhado para proporcionar uma estrutura a que podem ser adicionados outros critérios mais detalhados. Desta forma, desde sua concepção existem sugestões para que cada categoria possa ainda abranger mais aspectos.

Inclusive, as críticas que têm sido elaboradas à forma de avaliar questionam este fechamento em categorias. Em resposta a uma aparente separação e catalogação dos processos de experiência, existem também propostas vindas de uma tendência mais recente que propõe expandir o método para incluir metas não-conceituais, como por exemplo o "interesse" que é citado por Pekarik (2010: 107) como um aspecto não contemplado.

Outra crítica em relação a esta abordagem diz respeito aos resultados que produz. De acordo com McGregor (2008: 43), "the use of GLOs as a coding mechanism automatically leads the evaluator to attribute positive meanings to the evidence". Neste sentido, diz-se que cabe pouco questionamento em uma 
metodologia que se aproxima somente dos mecanismos de produção de discursos positivos a respeito dos processos de aprendizagem.

Um dos museus que faz uso dos GLOs é o National Museum of Scotland, onde as categorias da abordagem foram utilizadas para a construção de perguntas que foram feitas em entrevistas com os visitantes acerca de uma exposição. Jenna Fuchs (2007: 1), responsável pelo Serviço Educativo do Museu, apresenta a experiência onde, por meio das respostas dadas pelos visitantes, afirma que foi possível avaliar a transformação que aconteceu da sua aprendizagem e da sua experiência antes e depois da visita. Para Fuchs, a estrutura das cinco categorias permitiu que as diferentes leituras produzidas sobre os objetos emergissem durante a dinâmica de reflexão, uma vez que as questões respondiam a esta pluralidade.

Construindo um parecer sobre as características da abordagem, após a sua aplicação dentro dos procedimentos do National Museum of Scotland, Fuchs reflete sobre os GLOs:

The strengths of the GLOs are that they provide a common language to staff working together both within and across organizations, to talk about learning. They can help to steer projects so that the focus on the end user, i.e. the visitor, remains a priority. And subsequently, that can help to focus evaluation by ensuring that relevant questions are asked, thus giving a more accurate measure of the impact projects make on visitor learning in relation to desired learning outcomes (2007: 5)

Contudo, Fuchs (2007: 5) também sinaliza fragilidades nesta abordagem, argumentando que, sobretudo a categoria "habilidade", coloca os visitantes numa posição desconfortável, fazendo-os sentir-se testados. Outra questão levantada diz respeito ao tempo e de uma forma diferente das que já foram apontadas anteriormente, porque se refere ao momento onde a entrevista foi realizada e às dificuldades que se tem de pensar sobre o que acabou de acontecer, sem ter um momento para refletir sobre isso, sem haver distanciamento temporal para a reflexão.

Uma segunda abordagem de avaliação proposta aqui não está vinculada estritamente aos museus, mas dialoga com o que se tem defendido como posicionamento neste sentido. Trata-se da Assessment for Learning (AfL). Também desenvolvida no contexto do Reino Unido, este modelo criado por Paul Black e Dylan William (1998) relaciona-se com princípios discutidos pela avaliação formativa. De acordo com McGregor (2008: 41), as principais características dos AfL se relacionam com o efetivo questionamento, a criação de estratégias de feedback e retorno, a partilha dos resultados entre os pares e a autoavaliação. 
Centrada em um aspecto reflexivo, esta abordagem propõe uma autonomia dos estudantes, que gerem o controle de seu próprio processo de avaliação, criando mecanismos para repensar sobre sua aprendizagem. Neste sentido, os processos de reconstrução partem dos sujeitos e o controle está nos alunos, que posteriormente são também impulsionados a conversarem sobre a construção de sua avaliação e circularem o que foi entendido como autoavaliação.

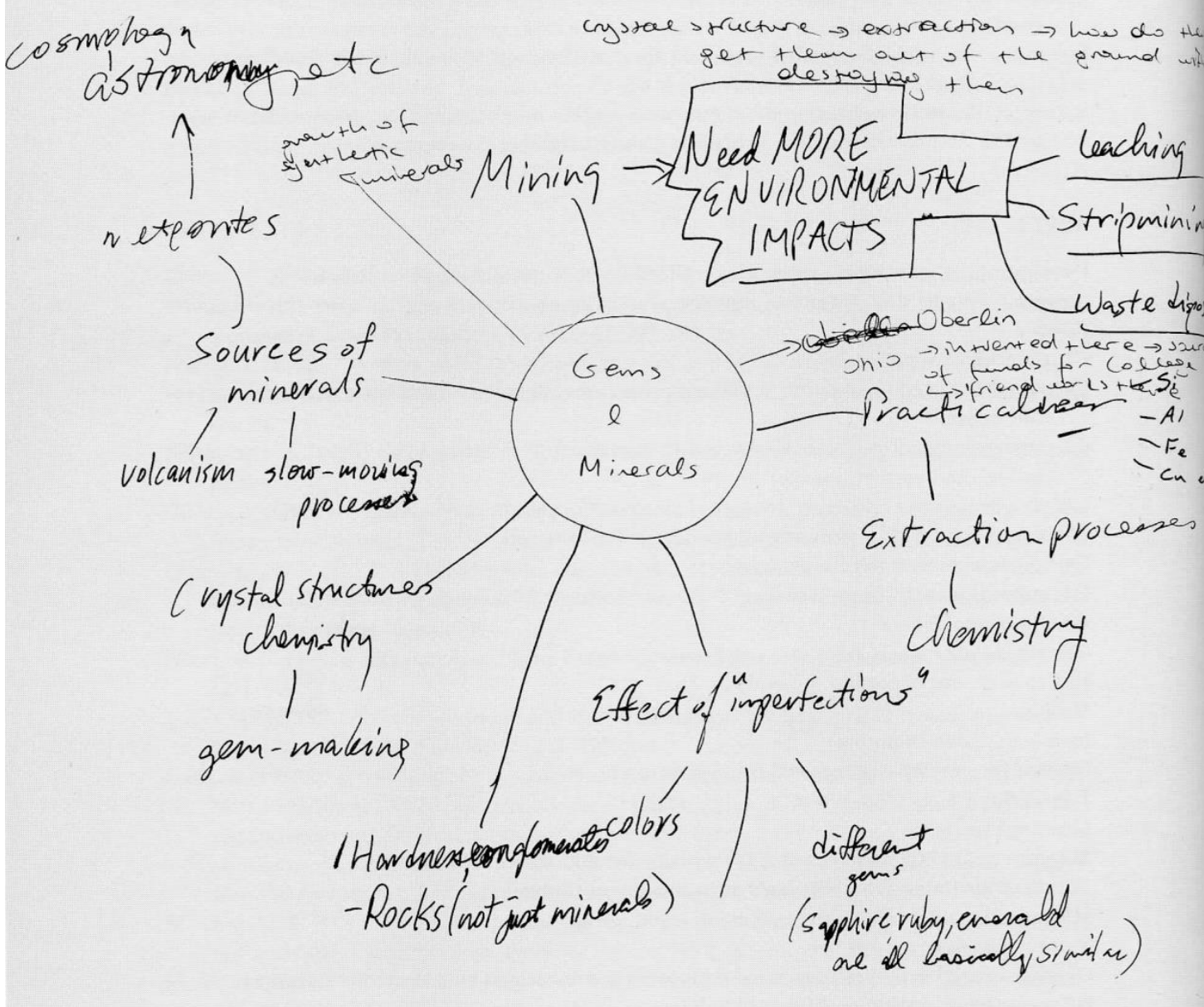

Fig. 2: Exemplo de aplicação dos Personal Meaning Maps. Fonte: FALK, John; MOUSSOURI, Theano; COULSON, Douglas. The effect of visitors' agendas on museum learning. Curator: The museum journal. Vol. 41, $n^{0} 2$ (1998), p. 107-120.

Assim, os alunos podem questionar sua própria aprendizagem e pensar de que forma existem criações de sentido a partir de suas próprias ideias. Por meio de uma abordagem processual que prevê um retorno constante, os alunos são impulsionados para resolver questões e formar ligações entre conceitos.

Para os professores, o modelo também se mostra como uma ferramenta reflexiva, uma vez que a responsabilidade de avaliação já não se assegura na 
relação direta entre os objetivos que determina e o cumprimento deles por parte dos estudantes. Nesta estrutura a prática de se pensar sobre as ações entra de forma mais relacionada com a investigação. Os professores são coparticipantes do processo de avaliação, que se refaz a todo momento na medida em que proposições também surgem por parte dos alunos.

Do ponto de vista da sala de aula, este modelo desenvolve no aluno a centralidade no seu processo de aprendizagem, dando autonomia e consciência para o que realiza enquanto estudante, impedindo-o de perder de vista seu objetivo ao longo do ano letivo. Dentro dos museus esta reflexão é mais direta e os estudantes são convocados a repensarem suas práticas no momento da visita.

Atuando em uma avaliação que seja para a aprendizagem e não sobre a aprendizagem, esta abordagem, quando deslocada para dentro dos museus, possibilita que haja uma consciência maior durante a avaliação, o que abre espaço para a criação dos próprios participantes para processos de desenvolvimento das estratégias de avaliação.

Outra estratégia para repensar as práticas são os Personal Meaning Maps (PMM), construída e discutida por John Falk, Theano Moussouri e Douglas Coulson (1998), e concebida para avaliar uma experiência de aprendizagem e tentar entender como cada indivíduo muda sua compreensão neste processo. Assim como nos GLOs, existem influências de teorias do construtivismo para a construção deste modelo, tal como situam os autores:

PMM utilizes this relativist-constructivist, as opposed to a positivistbehaviourist, approach to measuring learning. Specifically, this means it is assumed that each individual brings varied prior experiences and knowledge into a learning situation and that these shape how that individual perceives and processes what he or she experiences. (1998: 109)

A questão-chave dos PMM é a mudança, baseada na qualidade da experiência que se vive dentro do espaço museal. 0 modelo dos PMM não assume que todos os estudantes terão uma mesma experiência ou construção de aprendizagem, bem como não entende que exista uma resposta única ou correta. A ideia é medir como uma vivência dentro do museu afeta cada pessoa, compreendendo a construção dos repertórios individuais.

A chave deste modelo é incorporar a multidimensionalidade da aprendizagem, pensando em como o tempo de contato dentro do museu modifica o que existia antes deste acesso. Para Falk, Moussouri e Coulson esta diferenciação entre o que existia antes e o que é construído depois determinam o aprendizado: 
The combination of prior experience and the new experience result in learning, but the resulting learning is unique for each individual, situated within the contexto in which it was learned. Given the varied starting and ending point of each individual learner, traditional methods of assessment, which rely upon everyone starting at the same place and ending at a similar have serious flags (1998: 109).

De forma prática, esta abordagem propõe que os participantes anotem todas as palavras, ideias e pensamentos que possam ter a respeito das imagens, dos objetos ou do que se vai construindo ao longo da prática educativa na visita à exposição. Estes registros são seguidos por discussão e estímulo para que todos inscrevam seus pontos de vista, baseados na construção do seu repertório.

Um exemplo de criação de um PMM realizado para uma exposição sobre gemas e minerais em um museu de ciências ilustra a prática deste modelo. 0 conceito-chave que envolve a exposição é posto como ponto de partida, de onde saem as ramificações que determinam os conceitos que vão sendo delineados a partir do entendimento que se tem do tema em questão. Nesta construção de diferentes caminhos e apêndices para um conceito inicial vãose criando pontes e relações que desdobram o conceito, ganhando o que poderia ser nominado de camadas de leitura, remontando ao que foi discutido em capítulos anteriores.

Ao final do processo este exercício é repetido, buscando registrar dados que permitam a análise das mudanças ocorridas durante a visita no museu. Esta valorização dos registros prévios e posteriores dão voz aos participantes, como refere João Pedro Fróis (2008: 72) quando reforça que "A natureza dos mapas de significação 'refletem' a realidade cognitiva do sujeito e não a do investigador". Questiono, contudo, esta ideia, visto que o educador ou um investigador externo sempre interfere na forma como os processos se desenvolvem.

O mapeamento das construções de sentido oferece ferramentas para que os sujeitos reconheçam as modificações surgidas no seu repertório diante do diálogo desenvolvido no museu. Os conceitos, palavras, frases e imagens registrados passam a se relacionar, criando formas de contato que põem em diálogo ou confronto o que se fixou determinante antes e depois da experiência.

Como apontam Falk, Moussouri e Coulson (1998: 109) o intento dos PMM é o de comprovar que os museus conseguem afetar a aprendizagem dos sujeitos, mostrando por meio da comparação entre os conceitos, palavras e imagens empregados antes e depois da visita que o repertório dos visitantes sofreu alterações, sendo enriquecido depois do contato com os objetos do museu. 
Isso se averigua em aplicações do modelo, onde visitantes questionados sobre referências acerca de determinado assunto informam que ao final da experiência somaram interpretações às iniciais.

Este modelo aproxima-se de propostas que se constroem com base em análises diagnósticas que registram o que existia antes do contato com uma nova aprendizagem, que depois são utilizadas em comparação com novos registros da experiência. Considera-se, porém, que o objetivo não é averiguar melhoria ou evolução do que se sabe, porque não se pretende recair na postura de eficácia já questionada em relação à avaliação. $O$ propósito é identificar as mudanças que são construídas no contato - seja em relações de aproximação ou de confronto. A este respeito, John Falk e Lynn Dierking indicam:

Learning experiences successfully take into account the factor of prior knowledge, interests, and beliefs when they provide "hooks", or entry points, that enable learners to relate their previous experiences to this new one, be it new information being presented in an exhibition or program or an aesthetic connection being made through a special art gallery performance (2000: 182).

A prática educativa oferece estas aberturas e ganchos propostos, que atuam como impulso para que o visitante identifique em seu repertório novas pontes de contato, ampliando a ramificação e os caminhos criados nos mapas de criação de sentido que são produzidos. Dentro da mediação cultural, este modelo mistura-se entre uma proposta de repensar as práticas ao mesmo tempo em que atua como um objeto mediador da experiência.

Resultados práticos destes mapas de criação de sentido reconhecem que 0 repertório do visitante é mesmo ampliado e modificado durante a vivência dentro da exposição e isso não necessariamente se realiza vinculado a um exercício educativo. O próprio visitante pode identificar novas interpretações a partir de uma relação individual dentro do museu. A aplicação dos PMM comprova a ideia defendida por Falk e Dierking (1992) de que a experiência do visitante é afetada por eventos que modificam o repertório anterior e a forma como se relacionam com os objetos dos museus.

Uma última abordagem a ser apresentada é denominada Contemporary Gallery Education (CGE) e foi comissionada pelo Arts Council England em associação com a Engage 4 para descrever a relação dos públicos com a arte contemporânea e a partir disso constrói paradigmas em relação à reflexão deste processo. Segundo Emily Pringle (2006: 1), que concebeu os parâmetros

${ }^{4}$ Criada em 1989, a National Association for Gallery Education (NAGE) é hoje renomeada de Engage, uma Associação que é parte do Arts Council britânico e trabalha com mais de 1200 instituições entre museus, galerias e centros culturais em todo o Reino Unido. Propõe investigação, formações, eventos, publicações e documentos em torno da aprendizagem. 
deste modelo no livro Learning in the gallery, o objetivo desta abordagem é estimular a discussão e questões que pensem em processos de avaliação associados com espaços difusores da arte contemporânea.

O processo de ação, reflexão e experimentação que impulsiona a fabricação de arte sugere que, num quadro de aprendizagem vinculado ao CGE, 0 processo de participação é reconhecido tanto quanto qualquer outro resultado; é durante o tempo da ação que a reflexão ocorre. Para este modelo, a participação do artista dentro dos museus não se restringe aos aspectos produtivos, mas expande-se também para o trabalho educativo. Com o CGE, portanto, Pringle coloca a importância de atuação do artista como mediador cultural.

A presença dos artistas dentro do museu não é somente uma sugestão, neste caso, porque, de acordo com Pringle (2006: 14), esta aproximação é fundamental para a concretização de características fundamentais do CGE, tais como: o foco no processo de aprendizagem experencial e reflexivo como base; a centralidade do diálogo dentro da aprendizagem; a importância do questionamento e desenvolvimento do conhecimento, ao invés da busca por respostas; e a criação de comunidades de aprendizado, onde o conhecimento pode ser compartilhado e testado.

A caracterização desta abordagem extrapola a ideia de um método de avaliação, uma vez que ela é parte imbricada com o processo, onde os artistas promovem a participação dos visitantes para a discussão, troca de ideias e experiências. Com estes preceitos encontra-se reverberações entre o CGE com a ideia de mestre ignorante de Jacques Rancière (2002), onde neste caso o artista atuaria como um coaprendiz. Tal como McGregor (2008: 43) refere, "By focusing on where leaning happens, how it develops and what it involves, CGE stresses the factors that make learning in galleries, from works of art and (often) with artists, distinctive".

O processo é valorizado na colaboração e interação que acontece entre os sujeitos, propondo diálogos e partilhas dos conhecimentos e repertórios. A experimentação é valorizada como prática, que pretende ser questionadora no sentido de permitir reconstruções ao longo de um percurso sem um fim definido. De acordo com Pringle (2006: 7), "The model is characterised by experimental, open-ended, collaborative teaching and learning, and draws on a specific understanding os creative practice that can be identified as "conceptual'".

Esta abordagem se constrói com alguns pontos de contato com outros modelos já apresentados, tal como considera McGregor (2008: 43), quando diz que os CGE "has some overlap with the GLOs, but attaches more 
importance to observation and analysis of the context and process of learning", contudo na conceituação do modelo, Pringle (2006) se preocupa em referir que sua proposta é menos prescrita e mais descritiva, inclusive diferenciando-o dos GLOs; quando compreende que as categorias podem ser muito fixas, não abrindo espaço para o inesperado e para o emergente no fazer, tal como ocorre no CGE.

Para Pringle (2006: 30) existem ainda outras questões que diferenciam o CGE dos GLOs, uma vez que seu modelo propõe um processo de continuidade, enquanto os GLOs enfatizam em resultados para uma finalidade. Outra questão apontada diz respeito à passividade ao olhar para a "competência" categoria já questionada previamente - enquanto o processo que é defendido por Pringle busca o empoderamento.

Pensando neste modelo em comparação com os PMM, cabe dizer que o CGE não quer apenas compreender que mudanças ocorreram, mas também de que forma, com que complexidade e amplitude estas modificações foram construídas. Existe a preocupação de pensar na complexidade da experiência, de modo que ela não seja simplificada e tornada viável para análise. Sobre esta relação, McGregor (2008: 43) diz que os CGE também utilizam mapeamentos, contudo "so as to move away from constructions of learning as a process with clearly delineated endpoints or outcomes. It can also be used as a tool for both planning and evaluation".

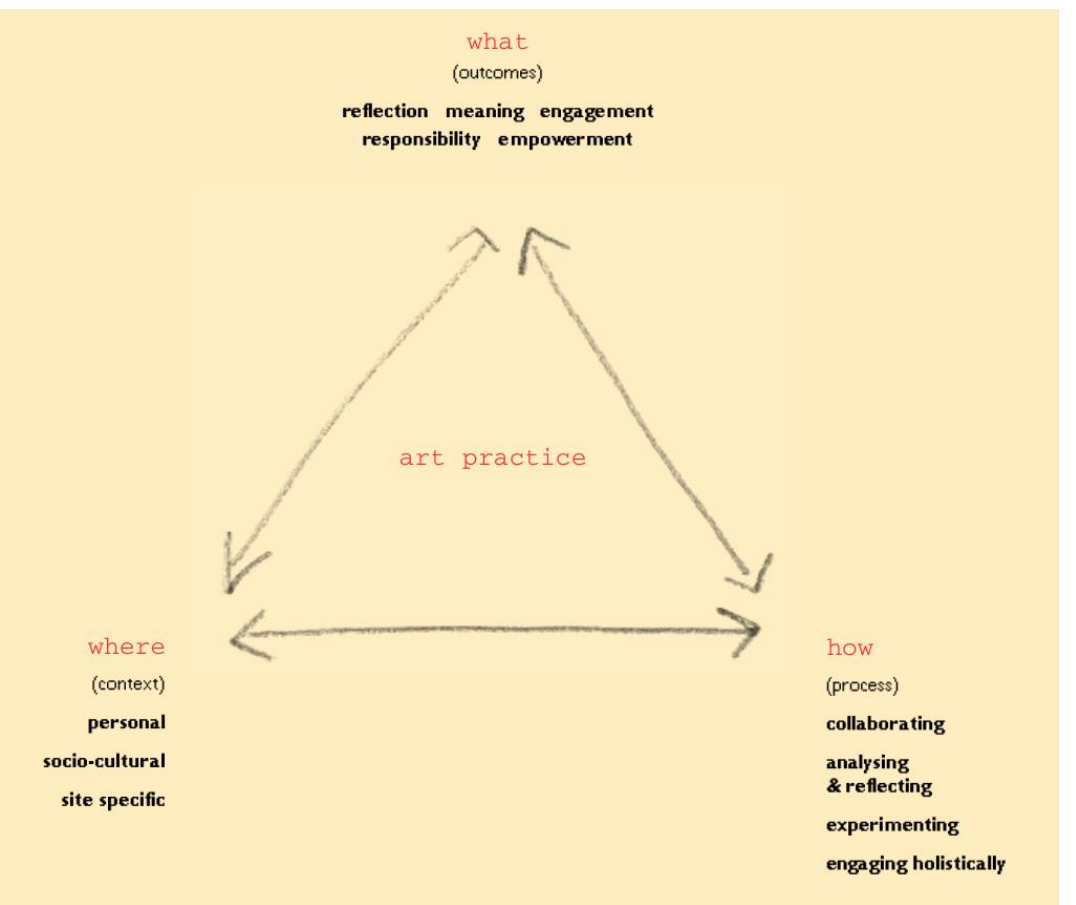

Fig. 3: Esquema do Contemporary Gallery Education. Fonte: PRINGLE, Emily. Learning in the gallery: context, process, outcomes. Londres: Engage, 2006 
O quadro apresentado acima é proposto como estratégia de ação para este modelo, que se mostra flexível o suficiente para acomodar o inesperado, o que vai sendo incorporado e desenvolvido nas trocas estabelecidas entre artistas e públicos. Pensando na prática artística como centro, três vértices se formam, constituindo o lugar onde a aprendizagem acontece (o contexto), como a aprendizagem se desenvolve (o processo) e o que a aprendizagem envolve (os resultados).

Por contexto se entendem as questões pessoais, que surgem do conhecimento prévio, da experiência e da motivação dos sujeitos. Também se pensa na constituição sociocultural da comunidade, do grupo que se forma e a relação com o educador artista. Por último, pensa-se nas relações estritamente ligadas ao lugar onde se realiza, apropriando o conceito artístico de site-specific para dentro dos processos de aprendizagem.

Os resultados são o outro vértice do triângulo que circunda 0 processo artístico, sendo realizado por meio das construções de sentido, do empoderamento dos discursos múltiplos que não se sobrepõem e pelo pensamento reflexivo que se articula para promover a aprendizagem. Novamente se reforça o quanto este modelo de avaliação não consegue somente propor estratégias de reflexão uma vez que suas características são baseadas na relação entre a ação (o fazer artístico, representado pela obra de arte exposta e também pelo fazer do educador artista) com a fundamentação que se faz deste processo prático.

Correndo o risco de ser redundante em relação ao que já foi apontado em outros modelos e na conceituação da avaliação reflexiva, uma problemática que envolve os CGE é o tempo, na medida que os exercícios de educação são entendidos como processuais e que não têm o começo ou o fim tão definidos, como sugere Pringle:

The CGE framework sees learning as part of an ongoing process. One of the difficulties associated with evaluating gallery education projects is that there can be a tendency to understand learning as having a finite beginning and end, which corresponds to the duration of the activity under investigation. This can be compounded by the exclusive use of tools such as entry and exit questionnaires to gather data (2006: 43).

A resistência ao uso de questionários convencionais - com questões fechadas e sem envolver aspectos reflexivos - é também apontada neste trecho, indicando que a recolha de registros não pretende ser fechada ou limitada a um tipo de respostas. A proposta se choca completamente com este tipo de abordagem, uma vez que nem pretende ter este encerramento definido, quanto mais utilizar um mecanismo que seja prontamente fechado. 
Com a apresentação destes diferentes modelos de avaliação que museus têm incorporado dentro de seu trabalho educativo identifica-se que a a associação de variados instrumentos permite uma leitura mais plural dos processos que são criados entre mediadores e públicos das instituições. A comunicação já existente entre os modelos evidencia o quanto os limites são tênues entre cada proposta.

A problemática do tempo é percebida como transversal a todos estes modelos, visto que o planejamento e a execução já demandam uma rotina de trabalho intensa por parte da equipe do museu. Ao entrar na etapa posterior, de análise dos materiais produzidos propriamente dita, o envolvimento de educadores, consultores e coordenadores precisa ser ainda mais intenso, evidenciando um contratempo para leituras críticas de todas as ações promovidas.

O que se sugere em resposta a esta problemática é que as investigações que atuem neste sentido de reflexão dos processos de recepção dos públicos adotem o procedimento de amostragem que recorrentemente é aliado às pesquisas acadêmicas. A análise de um recorte dos visitantes e das escolas que buscam o museu como meio para a educação possibilita um reconhecimento de fragilidades e potencialidades que podem ser trabalhadas no conjunto completo de públicos recebidos nos museus.

O trabalho mais individualizado confere uma possibilidade de enfrentar a questão do tempo e também responde a outra problemática apontada na análise destes modelos, que diz respeito ao caráter discursivo que todo procedimento avaliativo adota. A leitura a partir de um ponto de vista demanda um posicionamento, que além de ser político e impassível de neutralidade, também define apenas uma forma de ler o processo coletivo das visitas educativas.

Por isso que sugere-se a amostragem para conseguir desenvolver procedimentos de avaliação mais pontuais e focados na análise plural do que se realiza durante a mediação cultural. Refletir processualmente sobre experiências focais possibilita que os educadores e os públicos recorram a variadas ferramentas para avaliar as práticas educativas realizadas, analisando criticamente o que foi produzido e pensando em resposta aos objetivos pré-definidos.

Para além de associar múltiplos instrumentos para pensar a avaliação, com 0 levantamento destes quatro diferentes modelos indica-se que cada instituição identifique, nos seus encontros, a melhor forma de conseguir criar ferramentas de escuta para se comunicar com seus públicos. Ao invés de compreender estes modelos de forma taxativa, sugere-se a criação de novos preceitos a 
partir destas experiências que já estão em desenvolvimento, dando respostas e novas questões para os museus citados.

Desenvolver estratégias de avaliação para repensar os processos educativos pressupõe que as instituições devem responder uma série de perguntas visando identificar as motivações que envolvem a criação deste trabalho. Museus precisam pensar por quê querem avaliar, quem querem ouvir, que uso querem fazer dos resultados e de que forma o seu trabalho será modificado a partir das respostas que conseguirem. No conhecimento de modelos e ferramentas que já estejam funcionando no sentido da escuta e com estas respostas das intencionalidades de forma crítica e consciente, cada setor educativo desenvolverá seus próprios canais para refletir com os públicos visitantes.

\section{Referências}

BINKS, George; UZZELL, David. Monitoring and evaluation: the techniques. In: HOOPER-GREENHILL, Eilean. (org.). The educational role of the museums. Leicester: Routledge, 1999.

BLACK, Paul; WILLIAM, Dylan. Assessment and classroom learning. Assessment in education: Principles, policy \& practice, v. 5, n. 1, p. 7-74, 1998.

BULL, Phil. A begginer's guide of museum evaluation. In: HOOPER-GREENHILL, Eilean. (org.). The educational role of the museums. Leicester: Routledge, 1999.

CUTLER, Nancy. Evaluation. In: JOHNSON, A. et al. (orgs.). The museum educator's manual - Educators share sucessfull techniques. Lanham: AltaMira Press, 2009.

ECONOMOU, Maria. Evaluation strategies in the cultural sector: the case of the Kelvingrove Museum and Art Gallery on Glasgow. Journal Museum and Society, v. 2, n. 1, p. 30-46, 2004.

FALK, John; MOUSSOURI, Theano; COULSON, Douglas. The effect of visitors' agendas on museum learning. Curator: The Museum Journal, v. 41, n. 2, p. 107-120, 1998.

FRÓIS, João Pedro. Os Museus de Arte e a Educação: Discursos e Práticas Contemporâneas. 2008.

FUCHS, Jenna. "Generic Learning Outcomes" as a strategic tool for evaluation learning impact. ICOM-CECA Viena Austria 2007. Disponivel em: <http://www.jennifuc hs.com/sites/default/files/2007-08\%20ICOM-CECA\%20Research\%20Paper\%20\%20BTPW\%20(J\%20Fuchs).pdf>. Acesso em: 6 ago. 2015.

HEIN, George. Evaluation of museum programs and exhibits. In: HOOPERGREENHILL, Eilean. (org.). The educational role of the museums. Leicester: Routledge, 1999. 
HOOPER-GREENHILL, Eilean. Museum and their visitors. Leicester: Routledge, 1994.

HOOPER-GREENHILL, Eilean. Museum and Education: Purpose, pedagogy, performance. Leicester: Routledge, 2007.

JONES, Penny; ANSON, Libby; HITCHINS, Alex. (orgs.). Watch this space - Toolkit. Londres: Engage in the visual arts, 2008.

McGREGOR, Sheila. Are we talking the same language? Approaches to assessment in schools and gallery education. In: JONES, Penny; DALY, Eileen. (orgs.). Watch This Space: Galleries and schools in partnership. Londres: Engage in the visual arts, 2008.

PEKARIK, Andrew J. From knowing to not knowing: moving beyond "outcomes". Curator: The Museum Journal, v. 53, n. 1, p. 105-115, 2010.

PINTO, Julia Rocha. Processos avaliativos em mediação cultural: a postura reflexiva das ações educativas. Dissertação (Mestrado em Artes e Educação). São Paulo: Universidade Estadual Paulista - UNESP, 2012.

PINTO, Julia Rocha. Reflexões sobre o meio: O espaço entre a Escola e o Museu de Arte Contemporânea. Tese (Doutorado em Educação Artística). Porto: Universidade do Porto, 2015.

PRINGLE, Emily. Learning in the gallery: context, process, outcomes. Londres: Engage in the visual arts, 2006.

RANCIĖRE, Jacques. O mestre ignorante. Belo Horizonte: Autêntica, 2002. 